\title{
THE INTEGRAL FORM OF THE SCHUR INEQUALITY
}

\author{
Béla FINTA \\ George Emil Palade University of Medicine, Pharmacy, Sciences and Technology of Tîrgu Mureş \\ Gheorghe Marinescu Street, no. 38, 540139 Tîrgu Mureş, Romania \\ e-mail: bela.finta@umfst.ro
}

\begin{abstract}
The purpose of this paper is to show the integral form of the original Schur inequality and to give some applications.
\end{abstract}

Keywords: Schur's inequality, Riemann integral

\section{Introduction}

It is well known the original Schur inequality with four variables:

$$
\begin{gathered}
x^{t} \cdot(x-y) \cdot(x-z)+y^{t} \cdot(y-x)(y-z)+ \\
+z^{t} \cdot(z-x)(z-y) \geq 0
\end{gathered}
$$

where $x, y, z, t \geq 0$ are positive real numbers [1], [2].

In paper [3] we considered a Schur type inequality with three variables:

$$
x^{t} \cdot(x-y)+y^{t} \cdot(y-x) \geq 0,
$$

where $x, y, t \geq 0$ are positive real numbers. Because the inequality is symmetric in the variables $x, y$ without loss the generality we can suppose $x \geq y \geq 0$ so $x^{t} \geq y^{t} \geq 0$. Then the verification of the inequality is immediately

$$
\left(x^{t}-y^{t}\right) \cdot(x-y) \geq 0 .
$$

After in [3] we gave some generalizations and concrete applications of this inequality.

The main part of the results of this paper was presented at [4].

\section{Main part}

The original Schur inequality is for four variables:

$$
x^{t} \cdot(x-y) \cdot(x-z)+y^{t} \cdot(y-x) \cdot(y-z)+
$$

$$
+z^{t} \cdot(z-x) \cdot(z-y) \geq 0,
$$

where $x, y, z, t \geq 0$ are positive real numbers. Without loss the generality we can suppose $x \geq y \geq z \geq 0$. Then $z^{t} \cdot(z-x)(z-y) \geq 0$ aुnd

$$
\begin{aligned}
x^{t} \cdot & (x-y)(x-z)+y^{t} \cdot(y-x)(y-z)= \\
& =(x-y) \cdot\left[x^{t}(x-z)-y^{t}(y-z)\right] .
\end{aligned}
$$


But $x^{t} \geq y^{t} \geq 0$ and $x-z \geq y-z \geq 0$,

$$
x^{t} \cdot(x-z) \geq y^{t} \cdot(y-z) .
$$

Consecvently

$$
(x-y) \cdot\left[x^{t} \cdot(x-z)-y^{t} \cdot(y-z)\right] \geq 0 .
$$

Let $f, g, h:[a, b] \rightarrow[0,+\infty)$ be three continuous functions, where $a, b \in \mathbb{R}, a<b$. For $v \in[a, b]$ let $x=f(v) \geq 0, y=g(v) \geq 0$ and $z=h(v) \geq 0$. Then from (1) we obtain

$$
\begin{aligned}
& f^{t}(v) \cdot(f(v)-g(v)) \cdot(f(v)-h(v))+ \\
& +g^{t}(v) \cdot(g(v)-f(v)) \cdot(g(v)-h(v))+ \\
& +h^{t}(v) \cdot(h(v)-f(v)) \cdot(h(v)-g(v)) \geq 0 \\
& \quad \forall v \in[a, b] .
\end{aligned}
$$

Using the Riemann integral we get the integral form of the Schur inequality:

$$
\begin{gathered}
\int_{a}^{b} f^{t}(v) \cdot(f(v)-g(v)) \cdot(f(v)-h(v)) d v+ \\
+\int_{a}^{b} g^{t}(v) \cdot(g(v)-f(v)) \cdot(g(v)-h(v)) d v+ \\
+\int_{a}^{b} h^{t}(v) \cdot(h(v)-f(v)) \cdot(h(v)-g(v)) d v \geq 0
\end{gathered}
$$

Conversely for fixed $x, y, z \geq 0$ positive real numbers let us choose the continuous functions $f, g, h$ : $[a, b] \rightarrow[0,+\infty)$ such that $f(v)=x, g(v)=y$ and $h(v)=z$ for all $v \in[a, b]$. Then from (2) we deduce

$$
\begin{gathered}
\int_{a}^{b}\left[x^{t} \cdot(x-y) \cdot(x-z)+y^{t} \cdot(y-x)(y-z)+\right. \\
\left.+z^{t} \cdot(z-x)(z-y)\right] d v \geq 0
\end{gathered}
$$

i.e.

$$
\begin{gathered}
{\left[x^{t} \cdot(x-y) \cdot(x-z)+y^{t} \cdot(y-x)(y-z)+\right.} \\
\left.z^{t} \cdot(z-x)(z-y)\right] \cdot \int_{a}^{b} 1 d v \geq 0
\end{gathered}
$$

so we obtain (1).

The integral form (2) is equivalent with the following:

$$
\begin{aligned}
& \int_{a}^{b} f^{t+2}(v) d v+\int_{a}^{b} g^{t+2}(v) d v+\int_{a}^{b} h^{t+2}(v) d v+ \\
& +\int_{a}^{b} f^{t}(v) g(v) h(v) d v+\int_{a}^{b} g^{t}(v) f(v) h(v) d v+ \\
& +\int_{a}^{b} h^{t}(v) f(v) g(v) d v \geq \\
& \geq \int_{a}^{b} f^{t+1}(v) \cdot h(v) d v+\int_{a}^{b} f^{t+1}(v) \cdot g(v) d v+ \\
& +\int_{a}^{b} g^{t+1}(v) \cdot f(v) d v+\int_{a}^{b} g^{t+1}(v) \cdot h(v) d v+ \\
& +\int_{a}^{b} h^{t+1}(v) \cdot f(v) d v+\int_{a}^{b} h^{t+1}(v) \cdot g(v) d v .
\end{aligned}
$$

For $t=0$ we deduce:

$$
\begin{aligned}
& \int_{a}^{b} f^{2}(v) d v+\int_{a}^{b} g^{2}(v) d v+\int_{a}^{b} h^{2}(v) d v \geq \\
& \geq \int_{a}^{b} f(v) \cdot g(v) d v+\int_{a}^{b} f(v) \cdot h(v) d v+ \\
& +\int_{a}^{b} g(v) \cdot h(v) d v .
\end{aligned}
$$

This inequality can be proved by direct calculus.

For $t=1$ we can obtain the inequality:

$$
\begin{aligned}
& \int_{a}^{b} f^{3}(v) d v+\int_{a}^{b} g^{3}(v) d v+\int_{a}^{b} h^{3}(v) d v+ \\
& +3 \cdot \int_{a}^{b} f(v) \cdot g(v) \cdot h(v) d v \geq \\
& \geq \int_{a}^{b} f^{2}(v) g(v) d v+\int_{a}^{b} f(v) \cdot g^{2}(v) d v+ \\
& +\int_{a}^{b} g^{2}(v) h(v) d v+\int_{a}^{b} g(v) \cdot h^{2}(v) d v+ \\
& +\int_{a}^{b} f^{2}(v) \cdot h(v) d v+\int_{a}^{b} f(v) \cdot h^{2}(v) d v .
\end{aligned}
$$

For $t=n, n \in \mathbb{N}$ we get the inequality: 
Using the Riemann integral we deduce the inequality:

$$
\begin{aligned}
& \int_{a}^{b} f^{n+2}(v) d v+\int_{a}^{b} g^{n+2}(v) d v+\int_{a}^{b} h^{n+2}(v) d v+ \\
& +\int_{a}^{b} f^{n}(v) g(v) h(v) d v+\int_{a}^{b} g^{n}(v) f(v) h(v) d v+ \\
& +\int_{a}^{b} h^{n}(v) f(v) g(v) d v \geq \\
& \geq \int_{a}^{b} f^{n+1}(v) \cdot h(v) d v+\int_{a}^{b} f^{n+1}(v) \cdot g(v) d v+ \\
& +\int_{a}^{b} g^{n+1}(v) \cdot f(v) d v+\int_{a}^{b} g^{n+1}(v) \cdot h(v) d v+ \\
& +\int_{a}^{b} h^{n+1}(v) \cdot f(v) d v+\int_{a}^{b} h^{n+1}(v) \cdot g(v) d v .
\end{aligned}
$$

We can choose concrete functions $f, g$ and $h$.

We obtain a natural generalization of the Schur inequality, if we consider a monotone increasing function $\alpha:[0,+\infty) \rightarrow[0,+\infty)$

$$
\begin{gathered}
\alpha(x) \cdot(x-y)(x-z)+\alpha(y) \cdot(y-x)(y-z)+ \\
+\alpha(z) \cdot(z-x)(z-y) \geq 0
\end{gathered}
$$

For $\alpha:[0,+\infty) \rightarrow[0,+\infty), \alpha(x)=x^{t}$ we reobtain the Schur inequality for four variables (1). We can choose more generally the function $\alpha: \mathbb{R} \rightarrow[0,+\infty)$, where the function $\alpha$ is monotone increasing or convex, which is the result of Valentin Vornicu [2]. In this case $x, y, z \in \mathbb{R}$ and

$$
\begin{gathered}
\alpha(x) \cdot(x-y)(x-z)+\alpha(y) \cdot(y-x)(y-z)+ \\
+\alpha(z) \cdot(z-x)(z-y) \geq 0 .
\end{gathered}
$$

More generally let $\alpha: \mathbb{R} \rightarrow[0,+\infty)$ be a continuous function, monotone increasing or convex, and let $f, g, h:[a, b] \rightarrow \mathbb{R}$ be given continuous functions. We choose $v \in[a, b], x=f(v), y=g(v), z=h(v)$ and we obtain

$$
\begin{aligned}
& \alpha(f(v)) \cdot(f(v)-g(v))(f(v)-h(v))+ \\
& +\alpha(g(v)) \cdot(g(v))-f(v))(g(v)-h(v))+ \\
& +\alpha(h(v)) \cdot(h(v)-f(v))(h(v)-g(v)) \geq 0
\end{aligned}
$$

for all $v \in[a, b]$.

$$
\begin{aligned}
& \int_{a}^{b} \alpha(f(v)) \cdot(f(v)-g(v))(f(v)-h(v)) d v+ \\
& +\int_{a}^{b} \alpha(g(v)) \cdot(g(v)-f(v))(g(v)-h(v)) d v+ \\
& +\int_{a}^{b} \alpha(h(v)) \cdot(h(v)-f(v))(h(v)-g(v)) d v \geq 0,
\end{aligned}
$$

or

$$
\begin{aligned}
& \int_{a}^{b} \alpha(f(v)) \cdot f^{2}(v) d v+\int_{a}^{b} \alpha(g(v)) \cdot g^{2}(v) d v+ \\
& +\int_{a}^{b} \alpha(h(v)) \cdot h^{2}(v)+\int_{a}^{b} \alpha(f(v)) \cdot g(v) \cdot h(v) d v+ \\
& +\int_{a}^{b} \alpha(g(v)) \cdot f(v) \cdot h(v) d v+ \\
& +\int_{a}^{b} \alpha(h(v)) \cdot f(v) \cdot g(v) d v \geq
\end{aligned}
$$

$$
\begin{aligned}
& \geq \int_{a}^{b} \alpha(f(v)) \cdot f(v) \cdot g(v) d v+ \\
& +\int_{a}^{b} \alpha(f(v)) \cdot f(v) \cdot h(v) d v+ \\
& +\int_{a}^{b} \alpha(g(v)) \cdot g(v) \cdot f(v) d v+ \\
& +\int_{a}^{b} \alpha(g(v)) \cdot g(v) \cdot h(v) d v+ \\
& +\int_{a}^{b} \alpha(h(v)) \cdot h(v) \cdot f(v) d v+ \\
& +\int_{a}^{b} \alpha(h(v)) \cdot h(v) \cdot g(v) d v .
\end{aligned}
$$

Now we choose the functions $\alpha, f, g, h$ and we can obtain concrete examples of inequalities.

Example 1. [2] Prove for every $x, y, z>0$ that

$x y(y-z)(z-x)+y z(z-x)(x-y)+x z(x-y)(y-z) \leq 0$.

We divide this inequality with the expression $-(x y z)<$ 0 and we obtain the equivalent inequality:

$\frac{1}{x} \cdot(x-y)(x-z)+\frac{1}{y} \cdot(y-x)(y-z)+\frac{1}{z} \cdot(z-x)(z-y) \geq 0$.

Now we take the convex function $\alpha:(0,+\infty) \rightarrow$ $(0,+\infty), \alpha(x)=\frac{1}{x}$. According to the result of Vornicu, this inequality is true. For $0<a<b$ we consider 
the continuous functions $f, g, h:[a, b] \rightarrow(0,+\infty)$. For $v \in[a, b]$ let $x=f(v)>0, y=g(v)>0$, $z=h(v)>0$. Then we can deduce the following inequalities:

$$
\begin{aligned}
& \int_{a}^{b} f(v) g(v)(g(v)-h(v))(h(v)-f(v)) d v+ \\
& \left.+\int_{a}^{b} g(v) h(v)(h(v))-f(v)\right)(f(v)-g(v)) d v+ \\
& +\int_{a}^{b} f(v) h(v)(f(v)-g(v))(g(v)-h(v)) d v \leq 0
\end{aligned}
$$

and

$$
\begin{aligned}
& \int_{a}^{b} \frac{1}{f(v)} \cdot(f(v)-g(v))(f(v)-h(v)) d v+ \\
& +\int_{a}^{b} \frac{1}{g(v)} \cdot(g(v)-f(v))(g(v)-h(v)) d v+ \\
& +\int_{a}^{b} \frac{1}{h(v)} \cdot(h(v)-f(v))(h(v)-g(v)) d v \geq 0
\end{aligned}
$$

Example 2. [2] For every $x, y, z \in \mathbb{R}$ show the inequality:

$$
\begin{gathered}
x^{4}+y^{4}+z^{4}+x y z(x+y+z) \geq \\
\geq x^{3}(y+x)+y^{3}(z+x)+z^{3}(x+y) .
\end{gathered}
$$

This inequality is equivalent with the following inequality:

$x^{2}(x-y)(x-z)+y^{2}(y-x)(y-x)+z^{2}(z-x)(z-y) \geq 0$,

which is the Schur inequality for the convex function $\alpha: \mathbb{R} \rightarrow[0,+\infty), \alpha(x)=x^{2}$. Let $f, g, h: \mathbb{R} \rightarrow \mathbb{R}$ be continuous functions. The integral form will be:

$$
\begin{aligned}
& \int_{a}^{b} f^{4}(v) d v+\int_{a}^{b} g^{4}(v) d v+\int_{a}^{b} h^{4}(v) d v+ \\
& +\int_{a}^{b} f(v) g(v) h(v)(f(v)+g(v)+h(v)) d v \geq \\
& \geq \int_{a}^{b} f^{3}(v) \cdot(g(v)+h(v)) d v+ \\
& +\int_{a}^{b} g^{3}(v) \cdot(h(v)+f(v)) d v+ \\
& +\int_{a}^{b} h^{3}(v) \cdot(f(v)+g(v)) d v
\end{aligned}
$$

or

$$
\begin{aligned}
& \int_{a}^{b} f^{2}(v)(f(v)-g(v))(f(v)-h(v)) d v+ \\
& +\int_{a}^{b} g^{2}(v)(g(v)-f(v))(g(v)-h(v)) d v+ \\
& +\int_{a}^{b} h^{2}(v)(h(v)-f(v))(h(v)-g(v)) d v \geq 0 .
\end{aligned}
$$

Example 3. [2] Let us show that for every $x, y, z \geq 0$ we have:

$x^{2}(y+z-x)+y^{2}(z+x-y)+z^{2}(x+y-z) \leq 3 x y z$.

This inequality we transform in the following equivalent form:

$x(x-y)(x-z)+y(y-x)(y-x)+z \cdot(z-x)(z-y) \geq 0$,

which is exactly the Schur inequality for the function $\alpha:[0,+\infty) \rightarrow[0,+\infty), \alpha(x)=x$, which is monotone increasing or convex. Let $f, g, h: \mathbb{R} \rightarrow[0,+\infty)$ be continuous functions. The integral form will be:

$$
\begin{aligned}
& \int_{a}^{b} f^{2}(v)(g(v)+h(v)-f(v)) d v+ \\
& +\int_{a}^{b} g^{2}(v)(h(v)+f(v)-g(v)) d v+ \\
& +\int_{a}^{b} h^{2}(v)(f(v)+g(v)-h(v)) d v \leq \\
& \leq 3 \cdot \int_{a}^{b} f(v) g(v) h(v) d v .
\end{aligned}
$$

Now we can put particular functions $f, g$ and $h$.

Let us consider the function $\alpha: \mathbb{R} \rightarrow$ $[0,+\infty), \alpha(x)=x^{2 k}$, where $k \in \mathbb{N}$. Then $\alpha$ is a convex function on $\mathbb{R}$. So for every $a, b \in \mathbb{R}, a<b$ and for every continuous functions $f, g, h:[a, b] \rightarrow \mathbb{R}$ we have

$$
\begin{aligned}
& \int_{a}^{b} f^{2 k}(v) \cdot(f(v)-g(v)) \cdot(f(v)-h(v)) d v+ \\
& +\int_{a}^{b} g^{2 k}(v) \cdot(g(v)-f(v)) \cdot(g(v)-h(v)) d v+ \\
& +\int_{a}^{b} h^{2 k}(v) \cdot(h(v)-f(v)) \cdot(h(v)-g(v)) d v \geq 0 .
\end{aligned}
$$

Let us consider the function $\alpha:[0,+\infty) \rightarrow$ $[0,+\infty), \alpha(x)=x^{2 k+1}$, where $k \in \mathbb{N}$. Then $\alpha$ is a convex or monotone increasing function on $[0,+\infty)$. 
So for every $a, b \in[0,+\infty), a<b$ and for every continuous functions $f, g, h:[a, b] \rightarrow[0,+\infty)$ we have

$$
\begin{aligned}
& \int_{a}^{b} f^{2 k+1}(v) \cdot(f(v)-g(v)) \cdot(f(v)-h(v)) d v+ \\
& +\int_{a}^{b} g^{2 k+1}(v) \cdot(g(v)-f(v)) \cdot(g(v)-h(v)) d v+ \\
& +\int_{a}^{b} h^{2 k+1}(v) \cdot(h(v)-f(v)) \cdot(h(v)-g(v)) d v \geq 0 .
\end{aligned}
$$

Let us consider the function $\alpha: \mathbb{R} \rightarrow$ $(0,+\infty), \alpha(x)=c^{x}$, where $c \in \mathbb{R}$ and $c>1$. Then $\alpha$ is a convex or monotone increasing function on $\mathbb{R}$. So for every $a, b \in \mathbb{R}, a<b$ and for every continuous functions $f, g, h:[a, b] \rightarrow \mathbb{R}$ we have

$$
\begin{aligned}
& \int_{a}^{b} c^{f(v)} \cdot(f(v)-g(v)) \cdot(f(v)-h(v)) d v+ \\
& +\int_{a}^{b} c^{g(v)} \cdot(g(v)-f(v)) \cdot(g(v)-h(v)) d v+ \\
& +\int_{a}^{b} c^{h(v)} \cdot(h(v)-f(v)) \cdot(h(v)-g(v)) d v \geq 0 .
\end{aligned}
$$

Let us consider the function $\alpha: \mathbb{R} \rightarrow$ $(0,+\infty), \alpha(x)=c^{x}$, where $c \in \mathbb{R}$ and $c \in(0,1)$. Then $\alpha$ is a convex function on $\mathbb{R}$. So for every $a, b \in \mathbb{R}, a<$ $b$ and for every continuous functions $f, g, h:[a, b] \rightarrow \mathbb{R}$ we have

$$
\begin{aligned}
& \int_{a}^{b} c^{f(v)} \cdot(f(v)-g(v)) \cdot(f(v)-h(v)) d v+ \\
& +\int_{a}^{b} c^{g(v)} \cdot(g(v)-f(v)) \cdot(g(v)-h(v)) d v+ \\
& +\int_{a}^{b} c^{h(v)} \cdot(h(v)-f(v)) \cdot(h(v)-g(v)) d v \geq 0 .
\end{aligned}
$$

\section{Discussion and conclusion}

In the next paper we will consider the Schur type inequality with five, six and seven variables obtained by the author and we will give the integral form of these inequalities with applications.

\section{References}

[1] https://en.wikipedia.org/wiki/Schur

[2] Valentin Vornicu, (2003), Olimpiada de Matematică de la provocare la experienţă, Biblioteca Olimpiadelor de Matematică, Editura GIL, Zalău, ISBN 973-9417-09-4.

[3] Béla Finta, (2020), Integral form of an algebraic inequality, Acta Marisiensis. Seria Technologica, volume 17(XXXIV), no. 1, pag. 27-30, George Emil Palade University of Medicine, Pharmacy, Science and Technology of Targu-Mures, ISSN 2668-4217, ISSN-L 2668-4217.

[4] Béla Finta, (2019), Forme integrale ale inegalitătii lui Schur, presented at the Conferinta Didactica Matematicii, editia a XXXV-a, Universitatea Babes-Bolyai, Facultatea de Matematica si Informatica, Sincraiu de Mures, Romania. 